This is a postprint version of the following published document:

$\mathrm{Li}, \mathrm{Xi}$, et al. Innovations through 5G-Crosshaul applications. EuCNC 2016, "The dawn of 5G", Papers (of the) European Conference on Networks and Communications, 27-30 June 2016, Athens, Greece. IEEE, 2016, pp. 1-6

DOI: https://doi.org/10.1109/EuCNC.2016.7561067

(C)2016 by IEEE. Personal use of this material is permitted. Permission from IEEE must be obtained for all other uses, in any current or future media, including reprinting/republishing this material for advertising or promotional purposes, creating new collective works, for resale or redistribution to servers or lists, or reuse of any copyrighted component of this work in other works. 


\title{
Innovations Through 5G-Crosshaul Applications
}

\author{
$\mathrm{Xi} \mathrm{Li}^{1}$, Giada Landi², Jose Núñez-Martínez ${ }^{3}$, Ramon Casellas ${ }^{3}$, Sergio González ${ }^{4}$ Carla Fabiana Chiasserini ${ }^{5}$, \\ Jorge Rivas Sanchez ${ }^{6}$, Domenico Siracusa ${ }^{7}$, Leonardo Goratti ${ }^{7}$ David Jimenez $^{8}$, Luis M. Contreras ${ }^{9}$ \\ ${ }^{1}$ NEC, ${ }^{2}$ Nextworks, ${ }^{3}$ CTTC, ${ }^{4}$ University Carlos III of Madrid, ${ }^{5}$ Politecnico di Torino, ${ }^{6}$ ATOS, ${ }^{7}$ CREATE-NET, ${ }^{8}$ VISIONA, \\ ${ }^{9}$ Telefonica I+D
}

\begin{abstract}
A transport network for 5G envisions integrating the fronthaul and backhaul segments (namely 5G-Crosshaul) into a single transport network. This requires a fully integrated and unified management of fronthaul and backhaul resources in a sharable, scalable and flexible way. The integrated and unified management of the transport network resources follows the Software Defined Networking (SDN) principles of centralization, decoupling between control and data plane and application ecosystem. Innovation to enable context-aware resource management and to provide system-wide optimization of QoS, as well as energy related objectives, lies on defining key applications as logical decision entities to program the underlying network and packet forwarding behaviour. This paper presents a set of 5G-Crosshaul SDN applications with the scope of (i) managing the 5G-Crosshaul resources, including not only network but also computing and storage resources, and (ii) offering efficient media distribution and traffic offloading solutions. In order to develop these applications in a real system, we identify the interactions between the applications and the control plane. Based on these interactions, we propose a two-layer application plane and specify the requirements of the applications on the Northbound Interface (NBI) of the control plane.
\end{abstract}

Keywords-Fronthaul; Backhaul; Resource Managment; SDN/NFV; Optimization; Northbound Interface (NBI)

\section{INTRODUCTION}

The 5G-Crossshaul project aims at developing an adaptive and cost-efficient solution for the $5 \mathrm{G}$ transport network, integrating both fronthaul and backhaul segments. The envisioned solution requires a fully integrated and unified management of fronthaul/backhaul resources in a sharable, scalable and flexible way. The control and management of such an integrated transport network, namely 5G-Crosshaul, will be based on the SDN principles and architecture defined by the Open Networking Foundation (ONF) [1] and will adopt Network Function Virtualisation (NFV) [2] concepts and mechanisms. They will also be aligned with the ETSI Management and Orchestration (MANO) architecture [3] as a specific means to offer a subset of the aforementioned services of network, cloud and storage. Our intention is to leverage state-of-the-art SDN/NFV architectures and enhance them so as to maximize the compatibility and integration of the system design with the existing standard frameworks and reference specifications. The controller plane, namely the 5G-Crosshaul Control Infrastructure (XCI), is composed of a hierarchy of network and cloud controllers, together with orchestration and management entities for Virtual Network Functions (VNFs). The controllers will be in charge of allocating, controlling, and configuring resources within the underlying network, computing and storage infrastructure in order to offer services in an optimal and efficient manner. The XCI interacts with the data plane entities via a Southbound interface (SBI) and offers a Northbound Interface (NBI) through which the appropriate resource abstractions are exposed to applications. The innovations brought by these applications will enable a system-wide resource optimization of power consumption, infrastructure utilization and cost, to create QoS-enabled unified transport services between network endpoints, i.e., 5G Points of Attachment (5GPoAs) and core network elements.

This paper presents a set of 5G-Crosshaul applications that use the services offered by the XCI in order to extend and complement it, by providing transport services offering context-aware resource optimization to manage network, computing and storage resources operating physical or virtual resources and enabling infrastructure sharing and multitenancy. In order to effectively design these applications, their interactions with the Crosshaul control plane need to be properly defined, taking into account the applications requirements in terms of information to be collected and the required operations to be performed on the underlying infrastructure. These interactions represent the application requirements on the NBI of the control plane. They can be seen as the main services that the 5G-Crosshaul control plane has to expose to the applications through the NBI. Besides, the interactions between the applications are also analysed. To this end, a two-layer application plane is proposed in this work.

The rest of the paper is structured as follows. Section II introduces the concept of the 5G-Crosshaul architecture design including control and data plane, and further highlights the functional role of applications in various service use cases. Section III describes the selected 5G-Crosshaul applications individually, while their interaction is discussed in Section IV. Section $\mathrm{V}$ analyses the interactions between the application plane and the control plane, and based on that it derives the NBI requirements in terms of main services of the XCI that need to be exposed to the applications. Finally, Section VI presents our conclusions.

\section{5G-CROSSHAUL ARCHITECTURE CONCEPT}

\section{A. 5G-Crosshaul Architecture Concept}

Figure 1 illustrates the Crosshaul architecture concept, which has three layers [4]. The lowest layer corresponds to the overlay of all infrastructure layers. The middle layer represents one of the key concepts of the Crosshaul vision: the 
integration of the different technologies (for both fronthaul and backhaul) in a common packet-switched network based on technology abstraction, and a unified data and control plane.

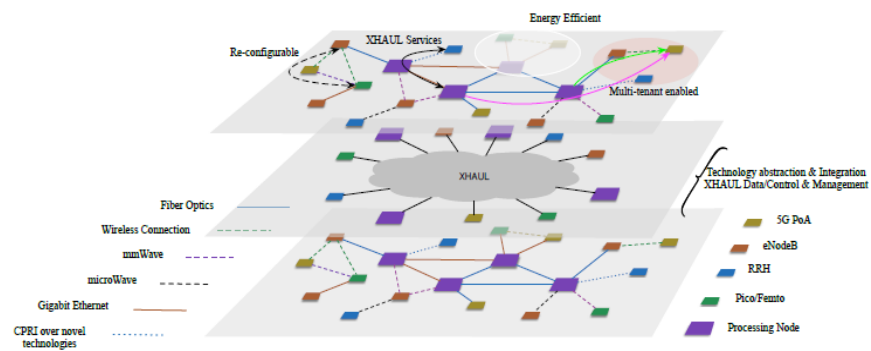

Figure 1: 5G-Crosshaul Architecture Concept

The uppermost layer presents the applications layer envisioned on top of the Crosshaul infrastructure in order to support the features related to:

- Re-configurability to cope with the level of demand expected from 5GPoAs and to allocate resources in a dynamic way, moving resources from areas where they are not needed to busier areas.

- Energy efficiency through techniques that reduce the energy consumption of the different Crosshaul elements, combined with the joint optimization of RAN and Crosshaul resources by dynamic de-activation or decommissioning of scarcely used network portions.

- Multi-tenant operation to enable a generalized sharing and more efficient utilization of the underlying resources, not only at the Crosshaul infrastructure level but also allowing operators and Over-the-Top (OTT) companies to quickly deploy services within the Crosshaul platform such as Video On Demand, high-definition video conferencing, TV broadcasting/multicasting, Content Delivery Networks (CDNs) and cloud services.

\section{B. 5G-Crosshaul Control Infrastructure (XCI)}

The design principle of the control plane is to support the different 5G-Crosshaul applications, which manage not only networking but also computing and storage resources, applied to both physical and/or virtual infrastructures. To do that, the control plane (i.e. the XCI) is designed to integrate the SDN principles control in the ETSI/NFV MANO architecture [3]. The SDN allows, based on a centralization of controllers, control and data plane separation and the specification of open and standard SBI enabling hardware programmability and deployment of modular applications. The NFV allows infrastructure and function virtualization which enables flexible and dynamic function placement (e.g. RAN functional split, mobility anchors) according to the transport network constraints and Service Level Agreements (SLAs). It also offers NFV services as defined by the ETSI NFV use cases, such as the deployment of Network Services (NS) like CDN, through the on-demand and automated instantiation of scalable VNFs interconnected through VNF forwarding graphs. The combination of both building blocks enables the service use cases described in Section II.D.
From a top-down perspective, the XCI is layered as follows. The first layer, NFV management and orchestration, includes the ETSI NFV MANO components such as the NFV orchestrator (NFVO), multiple VNF managers (VNFM), and the 5G-Crosshaul VIM extension referred to as the Virtual Infrastructure Manager and Planner (VIMaP, see section III.D). The second layer corresponds to the controller layer, composed of the network, computing, and storage controllers, in charge of managing the allocation and configuration of the different types of resources available in the NFV Infrastructure of a 5G-Crosshaul environment.

\section{5G-Crosshaul Data Plane}

The data plane needs to allow the integration of heterogeneous technologies for the fronthaul and backhaul links into a common packet based network. In the data plane, 5G-Crosshaul network Forwarding Elements (XFEs) interconnect a broad set of novel technologies building in effect a flexible packet-based network with high diversity. A unified, versatile frame format and the corresponding protocol suite will transport Crosshaul data over heterogeneous technologies that may span from fiber optics to wireless mmWave for instance. Crosshaul data plane is also comprised of 5G-Crosshaul Processing Units (XPUs) that are in charge of carrying out the bulk of operations in Crosshaul. These operations shall support C-RAN, thus hosting Base Band Unit (BBU), but also 5GPoA functionalities and a heterogeneous set of services (e.g. CDN-based services).

\section{Service Use Cases}

This section presents a high-level classification of the services that can be offered by the owner of the 5G-Crosshaul network to its clients. The classification is based on two main factors: the level of control offered to the customers on their virtual infrastructure and the tools used to provide services. More specifically, the infrastructure view that is exposed to clients may range from a single node to a complete description of networking, computing and storage resources of a virtual instance. The tools over which these services can be provided include the VNF templates that shall be handled by an NFV Orchestrator (NFVO), but also the APIs through which it will be possible to interact and control the physical and virtual resources of the infrastructure. To ease the identification, we have named the different cases according to the clients to which the services are offered: Over-the-Top (OTT), Mobile Virtual Network Operator (MVNO) and Mobile Network Operator (MNO).

- OTT Service Providers: This is the case in which service providers share the $5 \mathrm{G}$-Crosshaul network to reach their remote customers. This includes the case of a $\mathrm{CDN}$, as well as TV broadcasting/multicasting. In this use case, tenants of the 5G-Crosshaul operate in agnostic manner over the underlying infrastructure which they do not require to control directly. Among the three service use cases identified, this prescribes the mildest form of controlling Crosshaul resources. Indeed, in order to meet the QoS traffic requirements of the OTT, it is sufficient to provide resources in the form of computing power and storage, and routes allocated over the most adequate technologies, amongst the available ones. 
- MVNO: This use case considers virtual operators that act as providers of a virtual infrastructure, made available over the 5G-Crosshaul network. The MVNO uses the 5G Crosshaul infrastructure as a service without owing physical resources but only operating on the assigned virtual resources. This reflects the well-known situation of a virtual operator which serves its own customers, including OTT. Therefore, the MVNO requires access to and, to some extent, control of the virtual resources composing its own network, in order to manage and optimize them based on the traffic volume and the required level of QoS.

- MNO: This is the case of an operator which owns the 5GCrosshaul physical infrastructure to serve its own customers. In this specific situation, customers could be end users, as well as OTTs or MVNOs. The MNO can decide to lease resources to multiple MVNOs and/or directly allocate them to OTT providers. In order to assure consistency across the network and possibly reach optimization of resource utilization, the MNO has full access to and control of the Crosshaul resources.

\section{INNOVATIVE 5G-CROSSHAUL APPLICATIONS}

\section{A. Multi-Tenancy Application (MTA)}

Multi-Tenancy is a desired feature by 5G-Croshaul to enable a generalized, flexible sharing of 5G-Crosshaul infrastructures among multiple network operators or service providers (i.e., multiple tenants). The target is to significantly reduce the CapEx and OpEx by sharing the infrastructure resources and maximize their utilization in a cost-efficient manner. The 5G-Crosshaul XCI relies on the integration of and alignment with existing initiatives and projects (e.g., SDN controllers such as OpenDaylight [5]) supporting multitenancy to some degree. However, a coherent management of multi-tenancy is required horizontally, unifying the concepts of infrastructure virtualization and multi-tenancy in all involved segments and resources. For this purpose, the MultiTenancy Application (MTA) is needed to provide such management. The MTA is in charge of assembling these physical resources into a virtual network infrastructure and then allocate the virtual resources to the tenants. Each tenant is composed of a network subset with virtual nodes and links, referred to as a slice, owning a subset of the physical resources (including computing, storage, and networking resources). The tenant is created making use of virtualization techniques. The MTA allows on-demand, dynamic allocation of virtual resources to the tenants, providing per-tenant monitoring of network QoS and resource usage. Moreover, the MTA also allows the tenants to control and manage their own virtual resources. The main challenge is to ensure a clean isolation across tenants.

\section{B. Resource Management Application (RMA)}

Considering the high degree of flexibility which is required to provide network resources to service providers, MVNOs and MNOs, it is necessary to leverage on efficient resource management. This is indeed crucial in a shared multitenant environment to dynamically (re)allocate resources among several tenants. The RMA takes care of optimizing $5 \mathrm{G}-$
Crosshaul resources in centralized and automated fashion, in order to promptly react to network changes and, as discussed in Section II.C, to meet the requirements of different client applications. The RMA relies on the XCI controllers for the actual provision and allocation of resources. The RMA can operate over physical or virtual network resources, on a pernetwork or a per-tenant basis, respectively. Essentially, the RMA has two main functional pillars: (i) dynamic resource allocation and (re-) configuration (e.g., new routes or adaptation of physical parameters) as the demand and network state changes; and (ii) dynamic NFV placement, e.g., enabling multiple Cloud-RAN functional splits flexibly allocated across the transport network.

\section{Mobility Management Application (MMA)}

The main goal of MMA is to provide mobility management for mobility scenarios such as vehicle mobility use cases like high speed train scenarios, and also to optimize traffic offloading for media distribution like CDN, TV Broadcasting. The challenge for traffic offloading is to optimize the location and relocation procedures for services such as CDN in combination with resource management decisions. In this case, Crosshaul mobility will be based on a flat IP network, on which traffic is forwarded to the nearest point of connection to the Internet. The forwarding will be based on direct modification of flow tables at the data path elements, using, e.g., the OpenFlow protocol [6]. The MMA aims to provide traffic offload to the Internet and/or moving the applications to the edge as close as possible to the users. The MMA uses the services offered by the RMA to provide best paths between the different elements of the network, with the main goal to optimize the route or path followed by mobile users' traffic towards the Internet or to a core service provided in a datacenter. The assignment of Points of Connection (PoC) to the Internet and possible points to offload to $\mathrm{CDN}$ networks or core nodes will depend on the criteria adopted by each tenant owning the network. After computing the best set of elements to provide a service to the user, the MMA will request the RMA to find the best path connecting these points based on the network status. The focus of the MMA is to exploit the context information as well as the load of some candidate target Base Stations (BSs), in determining the target $\mathrm{BS}$ and the corresponding resource allocation. This application will exploit the deterministic trajectory of the node for the proactive creation of paths in advance, placing cache nodes and even core nodes in the path of movement.

\section{Virtual Infrastructure Manager and Planner (VIMaP)}

Initially conceived as a 5G-Crosshaul application, the Virtual Infrastructure Manager and Planning application (VIMaP) is now logically part of the 5G-Crosshaul XCI and resides at the lowest level of the application hierarchy. It extends the ETSI/NFV Virtual Infrastructure Manager (VIM) [2] by offering additional services and by implementing logic and policies for the optimal allocation and placement of resources in a pluggable and modular way. The VIMaP enables other applications (such as the MTA) or other XCI functional elements (e.g., the VNF manager) to request the constrained allocation of physical and virtual Crosshaul resources (i.e., computing and networking resources). The VIMaP implements the mechanisms to instantiate, deploy and 
provide these resources over the Crosshaul infrastructure. It acts as a bridge between an application requiring a heterogeneous set of resources (e.g., a slice) and the actual interaction with one or multiple underlying controllers. The VIMaP itself, consequently, exports an API to the aforementioned applications, and this API constitutes its NBI.

For planning purposes, both services (in the form of distributed VNFs across Crosshaul) and resources (in terms of computing capabilities hosting those VNFs and networking resources connecting them internally and externally to Crosshaul) have to be accounted for in a combined process of allocation, with the additional complexity of satisfying and consolidating demands coming from multiple tenants.

\section{E. Energy Management and Monitoring Application (EMMA)}

The Energy Management and Monitoring application (EMMA) is an infrastructure-related application of the 5GCrosshaul system. It aims at monitoring energy parameters of RAN, fronthaul and backhaul elements, estimate energy consumption and trigger reactions to optimize and minimize the energy footprint of the virtual network while maintaining the required QoS for each VNO or end user. Together with energy-specific parameters like power consumption and CPU loads, EMMA will also collect information about several network aspects: traffic routing paths, traffic load levels, user throughput and number of sessions, radio coverage, interference of radio resources, and equipment activation intervals. All these data can be used to compute a virtual infrastructure energy budget for subsequent analyses and optimizations.

The application is designed to optimally schedule the power operational states and the levels of power consumption of 5G-Crosshaul network nodes, jointly performing load balancing and frequency bandwidth assignment, in a highly heterogeneous environment. Also the re-allocation of virtual functions across 5G-Crosshaul will be done as part of the optimization actions. This will allow moving fronthaul or backhaul VNFs to less power-consuming or less loaded servers, thus reducing overall energy footprint of the network.

\section{F. CDN Management Application (CDNMA)}

The Content Delivery Network Management application (CDNMA) is an OTT application of 5G-Crosshaul related to the distribution of media content over $5 \mathrm{G}$ networks. Content distribution, especially video traffic, is expected to be the dominant contributor to the mobile data traffic demand. Thus, providing efficient ways of delivering content to the end users is a must. A CDN is a combination of a content-delivery infrastructure (in charge of delivering copies of content to endusers), a request routing infrastructure (which directs client requests to appropriate replica servers) and a distribution infrastructure (responsible for keeping an up-to-date view of the content stored in the $\mathrm{CDN}$ replica servers). This application is designed to manage the transport resources for a CDN infrastructure, controlling load balancing over several replica servers strategically placed at various locations to deal with massive content requests while improving content delivery based on efficient content routing across the 5G-
Crosshaul fronthaul and backhaul network segments and the corresponding user demands.

\section{G. TV Broadcast Application (TVBA)}

The Broadcast application (TVBA) aims to provide a solution for TV broadcasting \& multicasting services utilizing the 5G-Crosshaul architecture, running as an OTT service. A TV broadcasting/multicasting service is offered starting from the content of a live-source (e.g., a football match), which is processed till be finally transcoded to the objective format and bit rate (e.g., image resolution, scan format, etc.) and injected into the 5G-Crosshaul network. The TVBA deploys media transmission, live video broadcast over the 5G-Crosshaul infrastructure with focus on minimising both the cost and the spectrum consumption of the next generation TV. The TVBA offers broadcast as a service, taking the 5G-Crosshaul network as a facility for management of the construction, deployment and provision of the involved resources. The target is to optimize the content delivery and make sure a real-time delivery with the lowest possible delay is offered to the users.

\section{APPLICATION LAYER}

\section{A. Interaction Between Applications}

The possible interactions between the 5G-Crosshaul applications listed in the previous section are exemplified in Figure 2, which clearly shows that the application layer is divided into two sub-layers. The higher sub-layer includes OTT (Over-The-Top) applications, namely CDNMA, MMA and TVBA, which collaborate with one another and interact with lower sub-layer applications through their SBI. The lower sub-layer includes network-related applications: RMA, EMMA and MTA. The interactions among them are more complex than for the OTT apps, and go through the RMA, which also interacts with the upper sub-layer through its NBI. The EMMA only interacts with the RMA, providing support for the energy-efficient management of physical and virtual $5 \mathrm{G}-\mathrm{Crosshaul}$ resources. Interactions with the XCI are in large part delegated to the VIMaP and the MTA SBIs. Specifically, the MTA identifies a heterogeneous set of virtual resources (i.e., a slice) that the RMA demands and, in turn, it requests from the VIMaP the constrained allocation of physical and virtual 5G-Crosshaul resources. The VIMaP, located at the lowest level of the application hierarchy, proceeds to instantiate, deploy and provision such resources over the $5 \mathrm{G}$ Crosshaul infrastructure, interacting with one or multiple underlying controllers.

A number of use cases defined in 5G-Crosshaul will be supported through the usage of these applications. Network oriented functional services, such as multi-tenancy (relying on MTA) and Mobile Edge Computing (accessing RMA and MTA), will improve the efficiency and the utilization of the network, simplifying its operation and saving costs, thus reducing CapEx and OpEx, also in conjunction with energy optimization, as driven by EMMA. In addition to that, enduser oriented services like vehicle mobility (leveraging on MMA), media distribution (using CDNMA and TVBA) and dense urban society (mainly consuming RMA) will facilitate 
the introduction of new services in 5G-Crosshaul areas, thus generating new incomes to service providers. Finally, all of them will make use of VIMaP for infrastructure management.

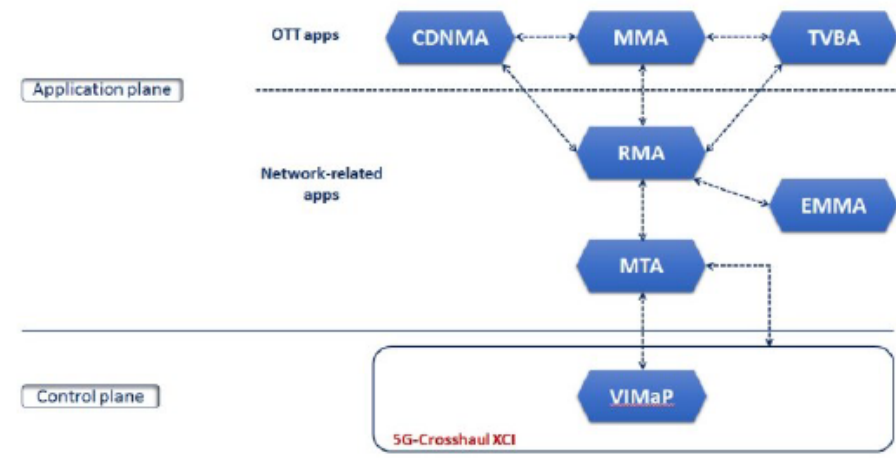

Figure 2: Interaction between applications

\section{B. Application Recursiveness}

This section details how the use of applications as functional entities (middleware) enables the construction of a recursive architecture that targets the multi-tenancy use case detailed before. Figure 3 shows the recursion and the layering of the XCI applications. In the lower layer we can find a Virtual Infrastructure Provider (VIP) who is also the owner of the physical infrastructure. In order to control this infrastructure, the VIP instantiates its XCI, fully compliant with the standard ETSI NFV architecture. The recursion is possible thanks to the coordination of the aforementioned applications in Section III: MTA, VIMaP and RMA. The VIMaP acts as an ETSI NFV VIM with planning features.

Tenants located on top of the VIP request a virtual infrastructure composed of a network subset with virtual nodes and links, i.e., a slice, to the MTA, which orchestrates the assignment of the available resources. The MTA requests to the VIMaP and the RMA the creation of a virtual topology with the resources demanded by the tenant; this virtual topology will be exposed to the tenant through the NBI.

Each tenant signs an SLA with the VIP. The provider must take care of the management of the physical infrastructure and must be sure to meet the requirements and provide the resources (networking, computing, storage) upon which they agreed. The control of the deployed virtual infrastructure is done through an API offered by MTA, with some defined operations and policies.

Tenants can operate on their virtual infrastructure as the VIP operates on the physical one, allocating and reselling part of the resources to other VNOs in a hidden way to the infrastructure provider (see Tenant\#1 with Tenant\#2 in Figure 3). This design provides a recursive hierarchical layering of the physical infrastructure offered to multiple tenants. The $\mathrm{XCI}$ can also provide control mechanisms to applications located outside the XCI through the NBI. The MMA, CDNMA and TVBA will make use of this interface. In case of deployment of network services like OTT tenants, where the service does not require recursion and multiple instantiations of the XCI, the API is offered through the NFV Orchestrator (NFVO).

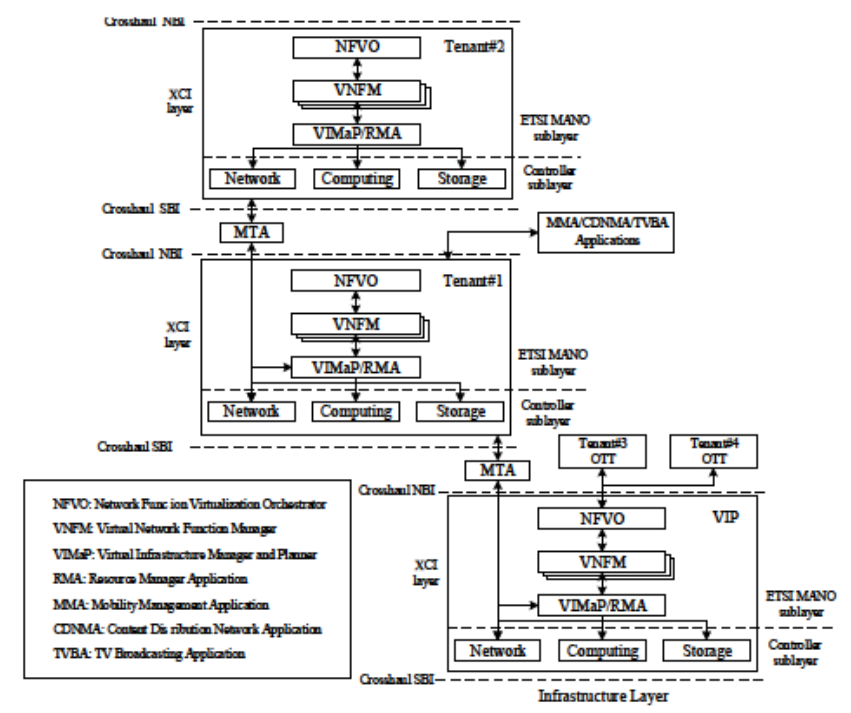

Figure 3: Recursion of XCI and layering of applications

\section{INTERACTIONS BETWEEN APPLICATION PLANE AND CONTROL PLANE}

\section{A. Interaction between Application and Control Plane}

The interaction between 5G-Crosshaul applications and $\mathrm{XCI}$ enables a dynamic and application-driven configuration of underlying Crosshaul infrastructures. In particular, the applications implement the service-dependent logic and the network intelligence to optimize resource usage and enable end-to-end service orchestration, while the XCI is responsible to enforce applications' decisions defined with a flexible level of abstraction and granularity.

The interaction of the XCI with the Crosshaul applications is done through the NBI. The NBI is composed of the APIs offered by the NFVO, and those offered directly by the underlying controllers and the VIMaP. The former API aims at supporting the OTT network services tenant, whereas the latter is oriented to support the MVNO and Network Operator-full access service use cases. Based on the kind of resource exposed, the XCI provides three types of NBI functionalities to Crosshaul applications, namely: i) network functionalities offered by the SDN controller, ii) computing and storage functionalities offered by the computing and storage controllers, and iii) unified IT/network service functionalities offered by the NFVO and the VIMaP, located in the MANO layer of the XCI.

The network side of Crosshaul infrastructures is controlled applying the SDN paradigms, through an NBI which follows the same principles of the Application-to-Controller-PlaneInterface (A-CPI) defined by the ONF standards [1]. This approach allows to abstract common network control concepts, like monitoring, provisioning of network connections or provisioning of isolated, multi-tenant, virtual network slices over a shared substrate. Thus, from the applications' point of view, all these services are accessible through a unified interface. The XCI gives a protocolindependent and technology-agnostic view of the underlying 
resources. The view for a tenant is restricted to the virtualization level and to the subset of (virtual) network elements associated to the given tenant.

At the upper layer of the NFV concept, the XCI through its MANO components allows the applications to operate on network services which are transparently deployed over a number of VNFs automatically allocated over 5G Crosshaul infrastructures. In this case, the XCI hides all the details of the joint configuration of network and IT elements and exposes a VNF-independent NBI to deploy, configure, manage and orchestrate entire network services (in the ETSI terminology) as well as their single components (i.e., the VNFs).

\section{B. NBI Requirements}

This section identifies and discusses the main XCI services, which are exposed to the 5G-Crosshaul applications through the NBI. In particular, these services can be classified in two main categories, namely informative and configuration services. Both kinds of NBI services can be exposed by APIs offered by the NFVO, VIM, and underlying controllers.

The informative services are used to collect data about resource availability, monitoring information, or unexpected events in the network and the IT domains of the underlying infrastructure. Depending on the type of information data and their usage at the application layer (e.g. input for medium/long term optimization or reactive and real-time decisions), polling or subscription/notification mechanisms should be implemented. This category includes the following services:

- Network topology service, to collect information about the physical network topology in terms of nodes, ports and links, with their characteristics, capabilities and resource availabilities.

- Mapping between VNF instances and the infrastructure resources.

- IT infrastructure inventory service, to provide a catalogue of computing and storage resources.

- Network monitoring service, to expose raw information collected by the physical network elements, depending on their monitoring capabilities.

- IT infrastructure monitoring service, to expose information related to the usage and performance of the underlying XPUs.

- Monitoring analytics service, to provide more elaborated information, like predictions, estimations of interference level or SNR, detected failures, etc.

The configuration services are used by the applications to request provisioning or operational actions for different kinds of resources. These services include:

- Provisioning of virtual infrastructures composed of VMs and virtual networks (IaaS model), to create multi-tenant infrastructure environments, which can be further used for deploying user-level applications.

- Management of templates and VNF packages for 5GCrosshaul OTT NSs.
- Provisioning of QoS-enabled network paths, to request customized network connections, with support for path hops specification, proactive scheduling and protection or restoration strategies.

- Enforcement of traffic scheduling, shaping and QoS provisioning, to regulate the traffic typically over already existing virtual infrastructures.

- Creation and management of single VNFs or end-to-end network services, to instantiate, monitor, configure and scale VNFs or Service Function Chains (SFCs).

- Configuration of network devices, enabling a low level access to network elements for a restricted set of commands (e.g., to regulate the power state of the devices for energy management purposes). This service provides the highest granularity of network programmability and it is usually consumed by applications dedicated to infrastructure optimization like the EMMA.

\section{CONCLUSIONS}

In this paper we presented the concept of the 5G-Crosshaul architecture that is based on the SDN/NFV principles. This architecture can manage network, compute, and storage resources of both physical and virtual infrastructures, flexible function placement, and deployment of network services as defined by ETSI. On top of that we defined a set of $5 \mathrm{G}$ Crosshaul applications, which are key innovations to enable context-aware resource management and to provide systemwide optimization of the integrated fronthaul/backhaul transport network for 5G. According to the functionalities of the applications and their interaction, a two-layer application plane structure was presented. Further, we analysed the interactions between the application plane and the control plane (i.e., XCI), and based on that we derived the requirements on the XCI NBI by defining a set of generic XCI services that need to be exposed to the applications.

\section{ACKNOWLEDGMENT}

This work has been supported by the H2020 project "5GCrosshaul: The 5G Integrated fronthaul/backhaul” (671598).

\section{REFERENCES}

[1] Open Networking Foundation, "SDN architecture - Issue 1 - ONF TR502", June 2014.

[2] "Network Functions Virtualisation - Introductory White Paper" (PDF). ETSI. 22 October 2012.

[3] "Network Functions Virtualisation (NFV); Management and Orchestration ETSI GS NFV-MAN 001 V1.1.1 (2014-12)".

[4] De La Oliva, A.; Costa Perez, X.; Azcorra, A.; Di Giglio, A.; Cavaliere, F.; Tiegelbekkers, D.; Lessmann, J.; Haustein, T.; Mourad, A.; Iovanna, P., "Xhaul: toward an integrated fronthaul/backhaul architecture in 5G networks," in Wireless Communications, IEEE, vol.22, no 5, pp.32-40, October 2015.

[5] Medved, J.; Tkacik, A.; Varga, R.; Gray, K., "OpenDaylight: Towards a Model-Driven SDN Controller architecture," in World of Wireless, Mobile and Multimedia Networks (WoWMoM), 2014 IEEE 15th International Symposium on a, vol., no., pp.1-6, 19-19 June 2014.

[6] Open Networking Foundation, "OpenFlow Switch Specification Version 1.4.0 - ONF TS-012”, October 2013. 